\title{
BMJ Open Postgraduate anaesthesiology education: protocol for a scoping review
}

\author{
Charlotte Ringsted, ${ }^{1}$ Sheena Bance, ${ }^{2,3}$ Laura Leigh Murgaski, ${ }^{4}$ Jodi Herold, ${ }^{4}$ \\ Susan Glover Takahashi ${ }^{4}$
}

To cite: Ringsted C, Bance S, Murgaski LL, et al.

Postgraduate anaesthesiology education: protocol for a scoping review. BMJ Open 2014;4:e004667. doi:10.1136/bmjopen-2013004667

\section{- Prepublication history for this paper is available online. To view these files please visit the journal online (http://dx.doi.org/10.1136/ bmjopen-2013-004667).}

Received 11 December 2013 Revised 22 April 2014 Accepted 25 April 2014

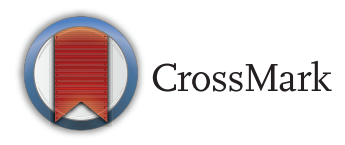

${ }^{1}$ Department of Anesthesia, The Wilson Centre, University of Toronto and University Health Network, Toronto, Ontario, Canada

2Department of Postgraduate Medical Education, Faculty of Medicine, University of Toronto, Toronto, Ontario, Canada

${ }^{3}$ Ontario Institute for Studies in Education (OISE), University of Toronto, Toronto, Ontario, Canada ${ }^{4}$ Post Graduate Medical Education, Faculty of Medicine, University of Toronto, Toronto, Canada

Correspondence to Dr Charlotte Ringsted; charlotte.ringsted@utoronto. ca

\section{ABSTRACT}

Introduction: The link between education and clinical practice is vital, yet the current state of research suggests there is a substantial gap between medical education research and practice. This too is the case in the domain of anaesthesiology education research, as much of the research focuses on simulation studies, and a narrow range of research methods. The aim of this study is to comprehensively review the existing literature in postgraduate anaesthesiology education research in order to identify key research priorities. The findings from this review will be used to establish a base for developing a strategic research programme in anaesthesia education and practice.

Methods and analysis: We will employ the scoping review methodology outlined by Arksey and 0'Malley (2005) to comprehensively search the literature pertaining to postgraduate anaesthesiology education. We will search relevant electronic databases (eg, MEDLINE, EMBASE) and grey literature. After conducting calibration exercises, two authors will independently apply inclusion criteria to all titles and abstracts and perform full-text review of all eligible articles. Data to be extracted will include basic information about the study (eg, location, design) as well as detailed information regarding the context of the research and type of education examined. Our results will be used to develop a framework of themes that outline the research currently being conducted and identify gaps in research.

Ethics and dissemination: This review is the first step in a strategic research plan in postgraduate anaesthesiology education. We plan to disseminate this research through publications, presentations and meetings with relevant stakeholders. Ethical approval was not sought for this scoping review.

\section{INTRODUCTION}

Anaesthesiology is one of the largest medical specialties, and research on anaesthesiology education and anaesthesiology practice continues to grow and diversify. Although the link between education and practice is crucial for patient safety and quality of care, it has been noted that a gap between education and clinical practice exists in the field of anaesthesiology. $^{1}$ A paucity of literature has

\section{Strengths and limitations of this study}

- The strength of this study is that it focuses on all aspects of clinical training.

- The limitation is that the study is confined to one specialty.

- A second limitation is that effect of educational interventions cannot be estimated - rather issues of studies on educational research will be compiled.

comprehensively surveyed what is currently known, and what is currently unknown from anaesthesiology education research. Philibert ${ }^{1}$ suggests that important topics in anaesthesiology that should be studied in more detail include issues surrounding transitions within anaesthesiology training, duty hour limits, and teaching and evaluating competencies (eg, the CanMEDS framework), and importantly, suggests that although important to knowledge and innovation, commentaries and editorials are often employed as an evidence base, ahead of alternative methodologies or article types. This concern is echoed by other researchers, who have noted a number of potential concerns with the current research, such as methodological issues (eg, small sample sizes, brief follow-up, threats to validity), ${ }^{1}$ lack of conceptual frameworks, ${ }^{2}$ and a preponderance of descriptive or justification studies (ie, those that focus on only the beginning or end of the scientific method, rather than employing all of the steps from making predictions through to testing them). ${ }^{3}$

A general overview of the existing literature suggests that much of the current research in anaesthesiology education is focused on technical skills, where simulation studies dominate the literature. ${ }^{4-6}$ However, an expert panel recently identified a number of areas within simulation-based training that need to be addressed in the future. ${ }^{7}$ Moreover, it appears that less is currently known about the clinical training environment, including important questions of clinical instruction and feedback, the structure of clinical 
training and developmental aspects of competence and training. ${ }^{8}$ A thorough understanding of the current evidence, as well as the consideration of what still needs to be examined, is essential to patient safety and quality of healthcare. However, at present, no study has systematically reviewed the literature in anaesthesiology education research to identify current practices, and the consequent gaps in current knowledge. To address this gap, we propose a comprehensive review of the literature pertaining to postgraduate anaesthesiology education. This review is the first step in a strategic research plan in postgraduate anaesthesiology education, and will seek to collect information on the methodologies and types of studies conducted, the conceptual or theoretical frameworks most often discussed (if any), the education components described, context of studies and articles and design. The overall purpose of this review will be to identify key research priorities in postgraduate anaesthesiology education to advance effective practice.

\section{METHODS}

A scoping review methodology will be used to conduct this study. Unlike systematic reviews, scoping studies/ reviews aim for breadth and comprehensiveness, rather than depth. ${ }^{9}$ Arksey and O'Malley's ${ }^{9}$ article outlining the framework for a scoping study is widely used in the planning and execution of scoping studies. The authors point to several reasons for conducting a scoping review, one being to identify research gaps in the existing literature. In particular, scoping studies are of use in areas in which little research has been conducted, as is the case in anaesthesiology education and research.

A protocol for our review was prepared and circulated to all team members, and will be revised as needed. We employed the five steps as outlined in Arksey and O'Malley's article (and further delineated by Levacet $a l^{10}$ ) to organise our review. These steps include (1) identifying the research question, (2) identifying relevant studies (developing the search strategy), (3) study selection, (4) charting the data (data extraction), and (5) collating, summarising and reporting the results.

\section{Data collection}

Search strategy

The basic structure of our search will include the terms 'postgraduate, 'anaesthesia'/'anaesthesiology' and 'education'. With the assistance of an information specialist, we will develop search strategies for the following databases: MEDLINE, EMBASE, ERIC and the Cochrane Database. We will also search reference lists of identified articles, hand search key journals (eg, Canadian Journal of Anesthesiology) and search reports (grey literature) from relevant organisations published from inception to December 2013, in order to identify other relevant information not captured by our search. We will not limit the search by study design or year, but due to a lack of resources for translation, will limit articles to those in
English. All references will be imported in RefWorks, an online bibliographic management programme, with duplicates removed.

\section{Study selection}

The inclusion criteria will include studies that mention anaesthesiology in some capacity, have a focus on the postgraduate level/residents and discuss education. More specifically we will include articles that mention anaesthesiology residents alongside other medical residents, in order to examine anaesthesiology residents' outcomes separately from other medical specialties. For specificity, however, we will only include articles that clearly mention postgraduate or a synonym for this level of training (ie, residents, house staff, intern etc). Finally, we conceptualise education as falling under the following categories: training, learning, teaching and assessment. We will use these terms to determine eligibility.

Prior to running the full database search, we will conduct a pilot calibration exercise with a random sample of 50 articles chosen from a MEDLINE search. Four authors (SGT, JH, LLM and SB) will independently screen the 50 articles for inclusion, using an excel sheet with headings for each inclusion criterion. We will compare the results between raters and determine the $\kappa$ coefficient. If the $\kappa$ is less than $90 \%$, we will repeat the calibration exercise as needed, and revise our inclusion criteria until reaching a $\mathrm{\kappa}$ of $90 \%$. We will then run the full database search and the full set of references will be independently screened for inclusion by title and reference by two authors (SB and LLM). Any discrepancies will be resolved through discussion with a third reviewer (SGT).

\section{Data extraction}

The full texts of all included articles will be retrieved. Two authors (SB and LLM) will complete the data extraction process independently. The data will be extracted via an online survey data collection tool, where data to be extracted will be entered as a question/answer in a survey. We chose this method in order to organise our data in a way that could be easily collated after being exported into Microsoft Excel. As suggested by Arksey and $\mathrm{O}^{\prime}$ Malley ${ }^{9}$ we will extract general information about each study/article, such as the author, year of publication, location, purpose, main findings or conclusions, and recommendations, suggestions, or implications as stated by the article. In addition, we will extract information specific to addressing the gaps in anaesthesiology education and research. This includes information about the topic of the article (eg, biomedical/technical studies), the conceptual or theoretical framework employed (eg, adult learning theory), the type of article (eg, review, commentary, primary research), the study design (if primary research study selected), the context of the study (eg, classroom; clinical) and the educational component (eg, assessment, teaching, training, including mode of delivery of teaching and training and assessment format; figure 1). Information on study design will be 
extracted using the 'research compass' framework as outlined by Ringsted et $a l^{11}$ which conceptualises medical education designs as experimental (eg, randomised control trial), explorative (eg, descriptive study), observational (eg, cohort study) and translational (eg, review or knowledge translation). Since we are including all study designs, including exploratory research and reviews which do not necessarily strive to provide evidence, we will not assess the quality of evidence (as recommended by Arksey and O'Malley ${ }^{9}$ ).

As with the title and abstract screening phase, we will first complete a calibration exercise for data extraction. Three authors (CR, LLM and SB) will, in duplicate, complete data extraction on a random sample of 10 articles that meet for inclusion in the title and abstract review. Using an online survey data collection tool, the results will be exported into Excel and compared using the $\kappa$ statistic. If the agreement level is sufficient, two authors will independently extract data from the full set of articles. If needed, a second calibration exercise will be conducted. The calibration exercises will be used for both refining the information to be extracted as well as improving the questions used in the online survey data collection tool. A third reviewer (CR) will adjudicate the calibration process in the case of discrepancies between reviewers.

\section{Synthesis and presentation of results}

We will use a mixed-method approach to summarise the data. We will provide a numerical summary of the included studies, including the overall number of studies, countries where studies/articles were conducted/written and years of publication. Next, we will summarise, using an iterative, content analysis approach, the gaps in postgraduate anaesthesiology education, following a grounded theory methodology. Specifically, the information extracted will be used to develop a framework of themes that outlines the research currently being conducted, and the potential gaps in research.

\section{DISCUSSION AND DISSEMINATION}

To the best of our knowledge, this study will be the first to review the literature on postgraduate anaesthesiology education using comprehensive scoping review methodology. This study is the first step in establishing an empirical base for developing a strategic research programme in anaesthesiology education and practice. The findings from the review will be submitted for publication in relevant medical education and anaesthesiology journals, which will provide information to researchers regarding topics and themes in postgraduate anaesthesiology education that are under-researched. The findings will also be disseminated at medical education conferences such as The International Conference on Residency Education (ICRE) and the Association for Medical Education in Europe (AMEE) Annual Conference. We will also share our results with key stakeholders in education, practice and collaborators.

Figure 1 Data extraction plan flowsheet. 
Acknowledgements The authors thank the PGME office at the University of Toronto for operational funding support and Laure Perrier for guidance and assistance with the search strategy.

Contributors CR conceived the study; all the authors worked collaboratively to draft and edit; all the authors read and approved the final manuscript.

Funding CR received support from a University of Toronto, Department of Anesthesia Merit Award for undertaking this project.

Competing interests None.

Provenance and peer review Not commissioned; externally peer reviewed.

Open Access This is an Open Access article distributed in accordance with the Creative Commons Attribution Non Commercial (CC BY-NC 3.0) license, which permits others to distribute, remix, adapt, build upon this work noncommercially, and license their derivative works on different terms, provided the original work is properly cited and the use is non-commercial. See: http:// creativecommons.org/licenses/by-nc/3.0/

\section{REFERENCES}

1. Philibert I. Review article: closing the research gap at the interface of learning and clinical practice. Can J Anesth 2012;59:203-12.
2. Boet S, Sharma S, Goldman J, et al. Review article: medical education research: an overview of methods. Can J Anesth 2012;59:159-70.

3. Cooke DA, Bordage G, Schmidt HG. Classifying the purpose of research. Med Educ 2008;42:128-33.

4. Bould MD, Naik VN, Hamstra SJ. Review article: new directions in medical education related to anesthesiology and perioperative medicine. Can J Anesth 2012;59:136-50.

5. Leblanc VR. Simulation in anesthesia: state of the science and looking forward. Can J Anesth 2012;59:193-202.

6. Boulet JR, Murray DJ. Simulation-based assessment in anesthesiology: requirements for practical implementation. Anesthesiology 2010;112:1041-52.

7. Issenberg SB, Ringsted C, Østergaard D, et al. Setting a research agenda for simulation-based healthcare education. A synthesis of the outcome from an Utstein style meeting. Sim Healthcare 2011;6:155-67.

8. Ringsted C. Developmental aspects of medical competency and training: issues of curriculum design. Med Educ 2010;45:12-16.

9. Arksey H, O'Malley L. Scoping studies: towards a methodological framework. Int J Soc Res Methodol 2005;8:19-32.

10. Levac D, Colquhoun H, O'Brien KK. Scoping studies: advancing the methodology. Implement Sci 2010;5:69.

11. Ringsted C, Hodges B, Scherpbier R. 'The research compass': an introduction to research in medical education: AMEE Guide No. 56. Med Teach 2011;33:695-709. 